\title{
Rapid screening of Aspergillus terreus mutants for overproduction of lovastatin
}

\author{
M.A. Vilches Ferrón ${ }^{1}$, J.L. Casas López ${ }^{1, *}$, J.A. Sánchez Pérez ${ }^{1}$, J.M. Fernández Sevilla ${ }^{1}$ and Y. Chisti $^{2}$ \\ ${ }^{1}$ Department of Chemical Engineering, University of Almeria, 04120 Almeria, Spain \\ ${ }^{2}$ Institute of Technology and Engineering, Massey University, Palmerston North, New Zealand \\ *Author for correspondence: Tel.: +34-950-015314; Fax: +34-950-015484; E-mail: jlcasas@ual.es
}

Received 26 February 2004; accepted 21 June 2004

Keywords: Aspergillus terreus, Candida albicans, lovastatin, mutation, screening

\section{Summary}

A novel rapid screening method is demonstrated for isolating lovastatin-overproducing strains of Aspergillus terreus. The screening methodology, based on the activity of lovastatin against the yeast Candida albicans, is nearly three times as fast as the selection methods used earlier. The new 6-h assay shows a linear correlation between the quantity of lovastatin generated by $A$. terreus isolates and the inhibition zones obtained on plates of $C$. albicans. The new technique is less expensive and requires less labour.

\section{Introduction}

Lovastatin is a potent cholesterol-lowering drug. Lovastatin acts by competitively inhibiting the enzyme 3hydroxy-3-methylglutaryl coenzyme A reductase (HMG-CoA) which catalyses the rate-limiting step of cholesterol biosynthesis. Lovastatin is produced as a secondary metabolite by a variety of filamentous fungi, including Penicillium sp. (Endo et al. 1976a), Monascus ruber (Endo et al. 1976b; Juzlova et al. 1996), and Aspergillus terreus (Alberts et al. 1980). Commercial production of lovastatin uses $A$. terreus batch fermentation which has been investigated extensively (Novak et al. 1997; Manzoni et al. 1998, 1999; Szakács et al. 1998; Kumar et al. 2000a).

Random mutation and selection strategies have been reported for obtaining overproducing isolates of $A$. terreus (Vinci et al. 1991). A particularly useful rapid screening method for identifying lovastatin overproducers was described by Kumar et al. (2000b). The method was based on the anti-fungal properties of the $\beta$ hydroxyacid form of lovastatin and its inhibitory effect on the mycelial fungus Neurospora crassa. This bioassay required $16-18 \mathrm{~h}$ of plate incubation to measure zones of inhibition.

This paper describes a new method for screening for overproduction of lovastatin. Activity of lovastatin against the yeast Candida albicans is used as the basis for this method. Zones of inhibition proportional to lovastatin concentration were generated on agar plates that had been previously inoculated with $C$. albicans. This new 6-h assay allowed relatively rapid identification of lovastatin-overproducing strains of A. terreus. A mutation-selection program was carried out with
A. terreus ATCC 20542. After two mutation runs, a 4fold enhancement in production of lovastatin was achieved compared to the original strain.

\section{Materials and methods}

\section{Microorganism and inoculation}

The fungus used was obtained from the American Type Culture Collection, as Aspergillus terreus ATCC 20542. A suspension of spores was prepared by washing petri dish (potato dextrose agar, PDA) cultures with a sterile aqueous solution of $2 \%$ Tween $^{\circledR} 20$. The spore concentration was determined spectrophotometrically at $360 \mathrm{~nm}$ by reference to a standard curve that had been made by direct counts (Coulter EPICS XL-MCL flow cytometer) of spores in suspension.

\section{Mutation with EMS}

Aspergillus terreus spores were exposed to methanesulphonic acid ethyl ester (EMS) at three concentrations $(0.15,0.3$ and $0.5 \mathrm{M})$ for four durations of exposure (30, $60,90$, and $120 \mathrm{~min})$. The spores were grown for 4 days on PDA slants. Colonies were collected in agar plugs (3 mm diameter $\times 7 \mathrm{~mm}$ height) when more than $90 \%$ mortality had been attained in a dish. Each agar plug was transferred to an Eppendorf tube containing $500 \mu \mathrm{l}$ of the lactose-yeast-extract medium and incubated at $28{ }^{\circ} \mathrm{C}$ for 5 days. This medium consisted of (per litre): $120 \mathrm{~g}$ lactose, $1.85 \mathrm{~g}$ yeast extract, $2 \mathrm{~g} \mathrm{KH}_{2} \mathrm{PO}_{4}, 0.52 \mathrm{~g}$ $\mathrm{MgSO}_{4} \cdot 7 \mathrm{H}_{2} \mathrm{O}, 0.40 \mathrm{~g} \mathrm{NaCl}, 2 \mathrm{mg} \mathrm{Fe}\left(\mathrm{NO}_{3}\right)_{3} \cdot 9 \mathrm{H}_{2} \mathrm{O}$, $1 \mathrm{mg} \mathrm{ZnSO} \cdot \mathrm{H}_{2} \mathrm{O}, 0.04 \mathrm{mg}$ biotin and $1 \mathrm{ml}$ of a trace 
element solution. The trace element solution contained (per 1 of solution): $\mathrm{Na}_{2} \mathrm{~B}_{4} \mathrm{O}_{7} \cdot 10 \mathrm{H}_{2} \mathrm{O}, 100 \mathrm{mg} ; \mathrm{MnCl}_{2} \cdot 4-$ $\mathrm{H}_{2} \mathrm{O}, 50 \mathrm{mg} ; \mathrm{Na}_{2} \mathrm{MoO}_{4} \cdot 2 \mathrm{H}_{2} \mathrm{O}, 50 \mathrm{mg}$ and $\mathrm{CuSO}_{4} \cdot 5-$ $\mathrm{H}_{2} \mathrm{O}, 250 \mathrm{mg}$. The $\mathrm{pH}$ of the medium was adjusted to 5.5 before sterilization. Spores were harvested and plated at a concentration of $10^{6}$ spores per $\mathrm{ml}$ on duplicate fresh petri dishes with the same medium that had been solidified with $15 \mathrm{~g}$ of agar/l. The plates were incubated for 7 days at $28^{\circ} \mathrm{C}$.

\section{Bioassay with Candida albicans}

After incubation, A. terreus colonies from one of the duplicate dishes (A) were isolated in agar plugs punched using a sterile $10-\mathrm{mm}$ stainless steel cork borer and transferred to a screw-capped test tube. One millilitre of ethyl acetate was added for lovastatin extraction at $50{ }^{\circ} \mathrm{C}$ (15 min; with vortex agitation at 2 min intervals). The lovastatin extract was recovered by centrifugation $(2800 \times g, 5 \mathrm{~min})$. The other slant (B) was stored at 4 $6^{\circ} \mathrm{C}$ for further culture.

Candida albicans was grown for $12 \mathrm{~h}$ on PDA dishes at $28{ }^{\circ} \mathrm{C}$, then harvested and transferred at a concentration of $5-7 \times 10^{2}$ cells per $\mathrm{ml}$ on fresh PDA slants. Fifty microliters of lovastatin extract were transferred onto a $6 \mathrm{~mm}$ diameter paper disk and placed on the surface of a $90 \mathrm{~mm}$ diameter C. albicans plate. The spacing between lovastatin impregnated disks on a plate was at least $15 \mathrm{~mm}$. Negative standard disks were prepared with ethyl acetate alone. Positive standard discs were made by impregnating the paper with $50 \mu$ of a solution of known concentration of lovastatin in ethyl acetate. The plates were incubated for $6 \mathrm{~h}$ and zones of inhibited growth were recorded. A large diameter of the inhibition zone indicated a high titre of lovastatin.

\section{Lovastatin analysis}

Lovastatin was quantified as its $\beta$-hydroxyacid form, by HPLC. Because the fungus secretes lovastatin in the $\beta$-hydroxyacid form, the assay eliminated the conversion step to the active lactone form of the drug. Using the $\beta$-hydroxyacid permitted rapid analysis because this form elutes earlier from a chromatography column than does the lactone form of lovastatin. Also, the $\beta$-hydroxyacid is quite stable in solution. HPLC was performed on a Beckman Ultrasphere ODS $(250 \times 4.6 \mathrm{~mm}$ I.D., $5 \mu \mathrm{m}$ support diameter) column. A diode array detector was used. The eluent was acetonitrile $/ 0.1 \%$ phosphoric acid (60:40, by vol). The eluent flow rate was $1.5 \mathrm{ml} / \mathrm{min}$. The detection wavelength was $238 \mathrm{~nm}$. The sample injection volume was $20 \mu \mathrm{l}$.

\section{Shake flask cultures}

All fermentations were carried out at $28{ }^{\circ} \mathrm{C}$ in $100 \mathrm{ml}$ shake flasks filled with $20 \mathrm{ml}$ of the medium and held on a rotary platform shaker (150 rev/min, $2.6 \mathrm{~cm}$ stroke). The culture medium used was the lactose-yeast-extract previ- ously described. The culture lasted up to 7 days. The flasks were inoculated with $1 \mathrm{ml}$ of a spore suspension which had been standardized to contain $10^{6}$ spores $/ \mathrm{ml}$.

\section{Results and discussion}

In submerged cultures of $C$. albicans, addition of the $\beta$ hydroxyacid form of lovastatin caused growth inhibition at lovastatin concentrations greater than $0.06 \mathrm{~g} / \mathrm{l}$. On solid medium, when a given amount of lovastatin was placed on the agar surface using a paper disk, zones of no growth were obtained on plates of C. albicans. The diameter of the inhibition zones correlated linearly (Figure. 1) with the quantity of lovastatin impregnated in the paper disc, as follows:

\section{Inhibition zone diameter $(\mathrm{mm})=$ $0.195 \times$ lovastatin dose $(\mu \mathrm{g})$}

The value of the slope in the above equation will depend on the nature of the agar gel. The slope will be lower for gels containing a high concentration of agar because the agar content is well known to affect the rate of diffusion in the gel. Preliminary experiments showed a $10-20 \%$ variation in the inhibition zone diameters for different batches of agar gel. Considering this, a positive standard with a given amount of $\beta$-hydroxyacid lovastatin was placed on each plate and the measured diameters of inhibition zones were normalized relative to this standard for comparing with the calibration curve shown in Figure 1.

After establishing the bioassay protocol, two twostage mutation runs were carried out starting from the parent strain (A. terreus, ATCC 20542). Although in routine work only the high-yielding isolates (plate B) would be cultured in a liquid medium to assess the lovastatin production in submerged fermentation, in this study all the isolates were grown in shake flasks to illustrate the feasibility of the proposed methodology.

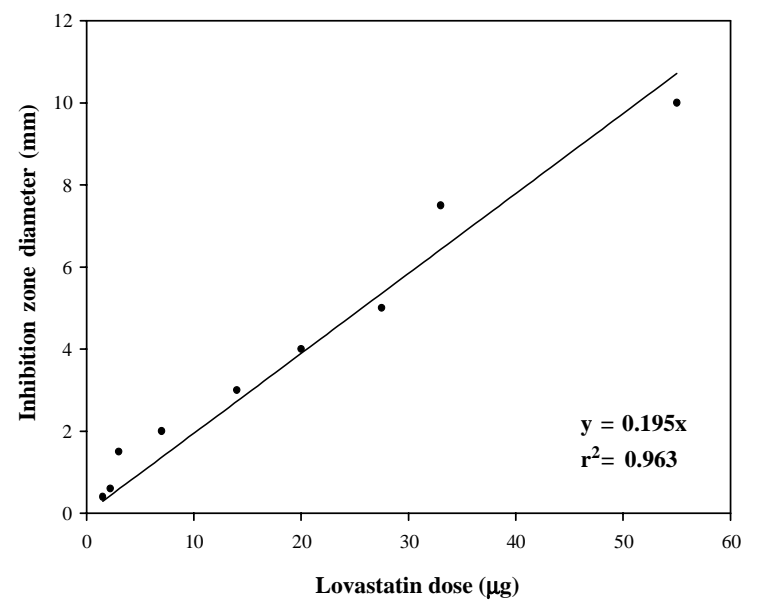

Figure 1. Standard curve for dose of the $\beta$-hydroxyacid form of lovastatin vs. inhibition zone diameter on $C$. albicans plates. 

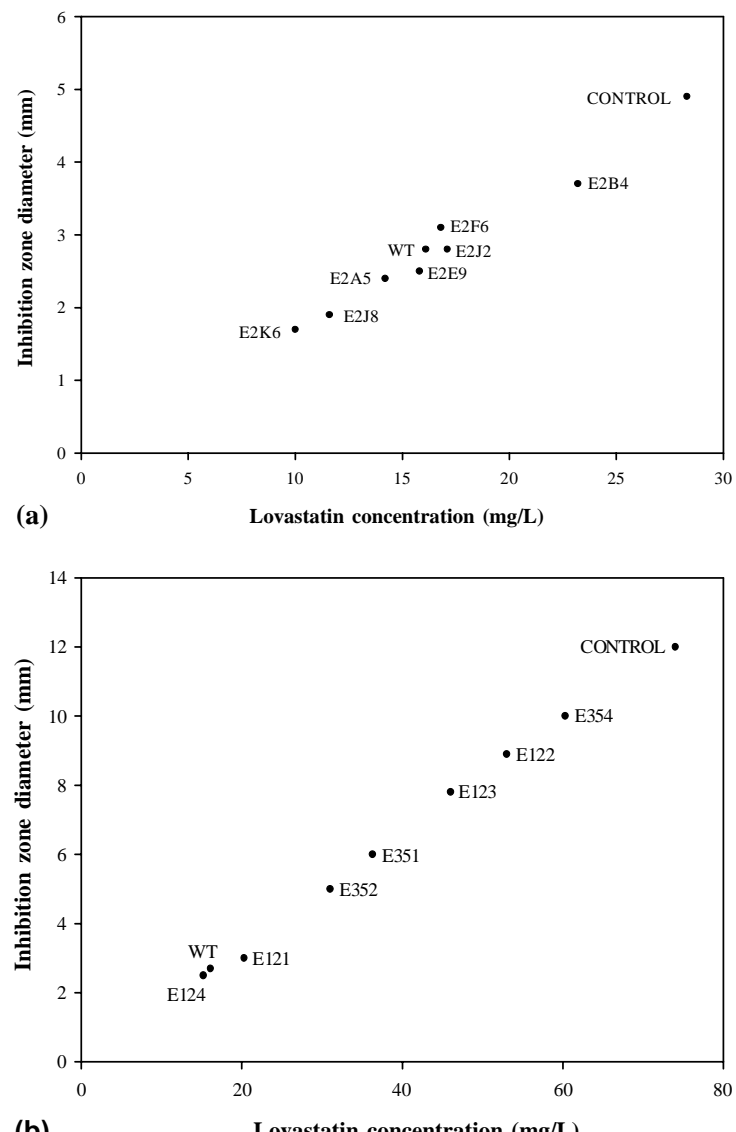

(b)

Lovastatin concentration $(\mathrm{mg} / \mathrm{L})$

Figure 2. Inhibition zone diameter on plates of $C$. albicans vs. lovastatin concentration (analysed by HPLC) from submerged fermentation of $A$. terreus isolates (duplicate cultures): (a) first stage mutation run; (b) second stage mutation run.

The first run of mutagenesis gave rise to mutants with a wide range of lovastatin production titres (Figure 2a). The parent culture displayed a lovastatin production level that was midway compared to the range for the isolates. There was a positive correlation (correlation coefficient $>0.98$ ) between the measured diameter of inhibition zone on agar (plate A) and the lovastatin titre obtained in liquid cultures of the isolate (plate B). The isolate E2B4 was selected for a second mutagenesis run (Figure 2b).

As expected, most of isolates from this second mutagenesis stage gave lovastatin titres higher than that of the parent culture (ATCC 20542). The highest titre $(60.3 \mathrm{mg} / \mathrm{l})$, obtained with the isolate $\mathrm{E} 354$, was four times the lovastatin production level of the original culture.

\section{Conclusions}

The screening methodology demonstrated in this study permitted isolation of lovastatin overproducer mutants of $A$. terreus in a significantly shorter period compared to the best existing methods. Also, the new technique was less expensive and required less labour. Currently, we have a substantial mutation/selection program underway for isolating better overproducer strains than those obtained already.

\section{Acknowledgement}

This research was supported by the Ministerio de Ciencia y Tecnología (MYCT) and FEDER (PPQ2000-0032-P402), Spain.

\section{References}

Alberts, A.W., Chen, J., Kuron, G., Hunt, V., Huff, J., Hoffman, C., Rothrock, J., Lopez, M., Joshua, H., Harris, E., Patchett, A., Monaghan, R., Currie, S., Stapley, E., Albers-Schonberg, G., Hensens, O., Hirshfield, J., Hoogsteen, K., Liesch, J. \& Springer, J. 1980 Mevinolin: a highly potent competitive inhibitor of hydroxymethylglutaryl-coenzyme A reductase and a cholesterol-lowering agent. Proceedings of the National Academy of Sciences of the USA 77, 3957-3961.

Endo, A., Kuroda, M. \& Tsujita, Y. 1976a ML-236 A, ML236 B and ML-236 C, new inhibitors of cholesterogenesis produced by Penicillium citrinum. Journal of Antibiotics 29, 13461348.

Endo, A., Kuroda, M. \& Tanazawa, K. 1976b Competitive inhibition of 3-hydroxy-3-methyl glutaryl coenzyme A reductase by ML-236 $\mathrm{A}$ and ML236 B fungal metabolites having hypocholesterolemic activity. FEBS Letters 72, 323-326.

Juzlova, P., Martinkova, L. \& Kren, V. 1996 Secondary metabolites of the fungus Monascus: a review. Journal of Industrial Microbiology 16, $163-170$.

Kumar, M.S., Jana, S.K., Senthil, V., Shashanka, V., Kumar, S.V. \& Sadhukhan, A.K. 2000a Repeated fed-batch process for improving lovastatin production. Process Biochemistry 36, 363-368.

Kumar, M.S., Kumar, P.M., Sarnaik, H.M. \& Sadhukhan, A.K. 2000b A rapid technique for screening of lovastatin-producing strains of Aspergillus terreus by agar plug and Neurospora crassa bioassay. Journal of Microbiological Methods 40: 99-104.

Manzoni, M., Rollini, M., Bergomi, S. \& Cavazzoni, V. 1998 Production and purification of statins from Aspergillus terreus strains. Biotechnology Techniques 12, 529-532.

Manzoni, M., Bergomi, S., Rollini, M. \& Cavazzoni, V. 1999 Production of statins by filamentous fungi. Biotechnology Letters 21, 253-257.

Novak, N., Gerdin, S. \& Berovic, M. 1997 Increased lovastatin formation by Aspergillus terreus using repeated fed-batch process. Biotechnology Letters 19, 947-948.

Szakács, G., Morovján, G. \& Tengerdy, R.P. 1998 Production of lovastatin by a wild strain of Aspergillus terreus. Biotechnology Letters 20, 411-415.

Vinci, V.A., Hoerner, T.D., Coffmann, A.D., Schimmel, T.G., Dabora, R.L., Kirpeker, A.C., Ruby, C.L. \& Stieber, R.W. 1991 Mutants of a lovastatin hyperproducing Aspergillus terreus deficient in the production of sulochrin. Journal of Industrial Microbiology 8, 113-120. 